\title{
LOS SMART CONTRACTS COMO ALTERNATIVA PARA LA MODERNIZACIÓN DE RECAUDACIÓN TRIBUTARIA EN ECUADOR ${ }^{\mathrm{I}}$ \\ Smart Contracts as an alternative for the modernization \\ of tax collection in Ecuador
}

\author{
Eugenia Novoa Z. ${ }^{2}$ \\ Cristina Escobar M. ${ }^{3}$ \\ Maria José Cajas A. \\ Ljubica Fuentes O.
}

Universidad Central del Ecuador, Quito, Ecuador

\begin{abstract}
Resumen
Si bien la administración tributaria, ha tenido varios avances en el aspecto de la digitalización de procesos, aún demuestra retrasos en su manejo de procesos frente a las necesidades que presenta una sociedad digital. Se ha evidenciado la necesidad de fomentar la implementación de tecnologías disruptivas que permitan desarrollar trámites de administración pública de forma transparente, descentralizada y eficiente. Esta necesidad se ha materializado justamente en la implementación de la plataforma de servicios como el "SRI en línea" o la sustanciación de trámites a través del sistema Quipux, como primeros intentos de digitalización de la administración. En el contexto nacional e internacional es preciso considerar como alternativa en mejora de la administración tributaria a los smart contracts y la tecnología blockchain.
\end{abstract}

\section{Palabras clave}

Blockchain, Contratos inteligentes, Tributación, Era digital, Administración pública.

\begin{abstract}
The tax administration in practice shows shortcomings in the face of the needs of a digital society. The need for technological means to carry out public administration procedures in a transparent, decentralized, and efficient manner has become evident. In the national and international context, it is necessary to consider smart contracts and blockchain technology as an alternative to the ineffectiveness of the tax administration.
\end{abstract}

\footnotetext{
${ }^{1}$ Las autoras de este artículo han contribuido en partes iguales.

${ }^{2}$ Master of Laws (LL.M). Docente. Universidad Central del Ecuador: Derecho cibernético, derecho financiero, derecho internacional. Coordinadora Fundadora de UNCTAD Youth Action Hubs Ecuador. CEO Digital Basis. Correo electrónico: pnovoa@uce.edu.ec; ORCID: 0000-0001-5098-6304.

3 Estudiante egresada Universidad Central del Ecuador. Asistente de cátedra e investigación Derecho Financiero. Vicecoordinadora de la Coalición Feminista Universitaria. Correo electrónico: ciescobar@uce.edu.ec; ORCID: 0000-00015970-9127.

${ }^{4}$ Estudiante Universidad Central del Ecuador. Asistente de cátedra e investigación Derecho Penal. Correo electrónico: mjcajasa@uce.edu.ec ORCID: 0000-0003-2975-5784.

${ }^{5}$ Estudiante Universidad Central del Ecuador. Asesora para la Sociedad Civil de ONU Mujeres. Coordinadora de la Coalición Feminista Universitaria. Vicecoordinadora de Youth Action Hubs Ecuador. Correo electrónico: 1mfuentes@uce. edu.ec; ORCID: 0000-0002-2831-6096.
} 


\section{Keywords}

Blockchain, Smart Contracts, Taxation, Digital Age, Public Administration.

\section{Introducción}

En el Ecuador la Constitución de la República fomenta un régimen tributario progresivo y transparente que dinamice la economía del país, mismo que debe estar a la par del contexto digital global. Si bien los principios constitucionales relativos al sistema tributario resultan alentadores, el desarrollo de los trámites la administración tributaria demuestra falencias lamentables frente a las necesidades que presenta una sociedad digital, falencias que pueden ser superadas a través de la implementación de tecnologías disruptivas.

En el contexto nacional e internacional es preciso considerar como alternativa en mejora de la administración tributaria a los smart contracts y la tecnología blockchain. La tecnología blockchain, que en rasgos generales es un sistema en el que cada entrada de información genera un "bloque", misma que se va enlazando y cifrando cada vez que se crea. En otras palabras, es una base de datos distribuida y segura. Esta tecnología disruptiva, posibilita la absoluta transparencia en el desarrollo de trámites, gracias a un sistema de validación descentralizado.

Por su lado, los smart contracts posibilitan desarrollar acuerdos convenidos por las partes mediante código fuente, estableciendo funciones específicas para que estos se ejecuten de forma automatizada.

Las soluciones que presentan los smart contracts en la era digital son inmensas, es preciso entonces, considerar su implementación en la administración pública y recaudación de tributos. En el contexto COVID -19 la sociedad ha evidenciado la necesidad de contar con medios tecnológicos que permitan desarrollar trámites de administración pública de forma transparente, descentralizada y eficiente.

El presente artículo comienza por presentar a los smart contracts, explicando su operatividad en materia tributaria. Se continuará por contextualizar al régimen tributario central del Ecuador. Finalmente, el artículo busca poner en evidencia la factibilidad de modernizar la administración tributaria central del Ecuador por medio de smart contracts y distributed ledger tecnologies o sistemas blockchain.

\section{Los smart contracts y su operatividad en materia tributaria}

El término smart contracts o "contratos inteligentes" hace referencia a un mecanismo automático y computarizado que permite la ejecución de instrucciones informáticas autoejecutables (Deloitte, 2017). Gracias a los smart contracts ahora es posible desarrollar acuerdos convenidos por las partes mediante código fuente, estableciendo funciones específicas para que estos se ejecuten de forma automatizada. Este tipo de contratos se codifican en sistemas descentralizados de blockchain, lo que los vuelve verificables y transparentes, eximiendo la necesidad de un intermediario. Las soluciones que presentan los smart contracts en la era digital son inmensas, particularmente explicaremos cómo estos optimizan la administración pública y recaudación de tributos.

\subsection{Contextualización de los smarts contracts}

Los smart contracts son la evidencia más palpable de la revolución digital en ejercicio de las relaciones jurídicas actuales. Se define a un smart contract como "un acuerdo legal que contiene o existe en forma de algoritmo" evidentemente, esto revoluciona la concepción previa de un contrato. En los contratos tradicionales sus términos y condiciones se establecen 
materialmente para, posteriormente, ejecutar el negocio jurídico. En la era digital, un smart contract ejecuta automáticamente parte o la totalidad del acuerdo por medio de medios digitales (Giuffrida, 2018, p. 760).

Debemos también tener en cuenta la diferencia entre un contrato inteligente y un contrato legal inteligente. El primero es el género, y se refiere a aquellos términos y condiciones previamente especificadas que se ejecutan automáticamente mediante un código de computadora, los mismos son almacenados en una cadena de blockchain. Por otro lado, un contrato legal inteligente es aquel creado y codificado para acoplarse a una normativa vigente, es decir, además de contener el acuerdo entre partes, cumple con la legislación que lo rige. (Digital Chamber of Commerce, 2018, p. 12)

Los contratos inteligentes se presentan en dos formas dependiendo su impacto o capacidad de ser modificados se clasifican en: contratos inteligentes débiles o weak smart contracts y contratos inteligentes fuertes o strong smart contracts. Los contratos inteligentes débiles son aquellos que, por orden de un juez, han podido ser modificados sin un costo alto después de su ejecución, por ejemplo, los contratos inteligentes que se realizan al pagar los parqueaderos públicos en las maquinas, que si bien no traen mayores complicaciones jurídicas retrotraerlos o cambiarlos no necesitarían un nuevo proceso de programación o uno muy complicado para dichos cambios. Por otro lado, los contratos inteligentes fuertes presentan costos relativamente más altos para ser alterados o revocados, por tanto, se dificulta su modificación, es decir su programación o seguridad implican que los cambios que se vayan a realizar implican una nueva programación y sistematización, por ejemplo, un contrato de compraventa de un bien inmueble que se inscriba mediante sistema blockchain (Raskin, 2017). Esta forma de contratación digital presenta mecanismos de verificación de transacciones eficaces, accesibles y seguros. Gracias a su codificación, se permite conectar de forma directa la información entre "peer-to-peer" o "P2P", lo que facilita el envío de datos de un servidor a otro en tiempo real (Deloitte, 2017, p. 6). La eliminación de intermediarios es una característica primordial de la contratación inteligente, ya que no es preciso contar con la intervención de otra persona que no sean las partes que conforman el contrato; es decir, se releva la necesidad de un notario, o un tercero que verifique la autenticidad de la transacción. (Meeting $\&$ Series, 2017). Ejemplo de esto en la administración tributaria, cuando el sujeto activo realiza una acción que implica un hecho generador, se genera la obligación de manera directa en el sistema de SRI por lo que ya estarían determinadas las imposiciones sin necesidad de trámites externos. En términos general, el proceso de los Smart contracts se resumen en la figura número 1.

\section{¿Cómo funcionan los contratos inteligentes?}

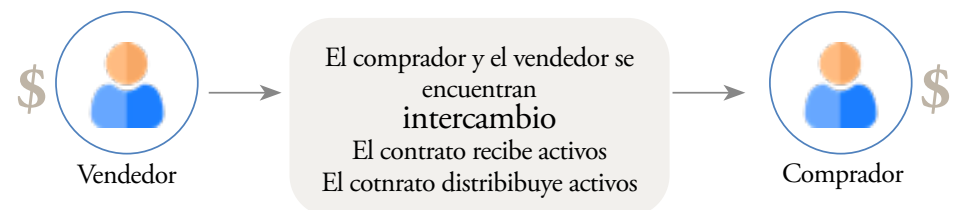

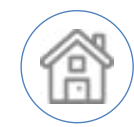

El derecho de propiedad es innegable

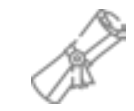

El acuerdo es automatizado

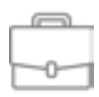

Registro

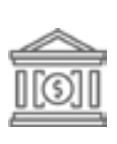

La confirmación de propiedad se produce de forma automática

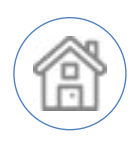

Digitalización del dinero

Figura 1. Smart contracts (Ameer, 2016, p. 1) 
Este sistema de contratos inteligentes, que no requieren la comparecencia física de las partes contratantes, bastaría con sus firmas electrónicas a través de tokens. Para implementar un sistema de blockchain con contratos inteligentes en Ecuador, el régimen legal necesitará especificar la figura de los contratos inteligentes, siendo necesarias reformas al marco regulatorio en materia contractual civil y comercial. El avance con respecto al sistema tradicional de contratación se debe a que los smart contracts se ejecutan por medio de blockchain o distributed ledger technologies, conforme se explica en los siguientes párrafos.

\subsection{Smart contracts en el blockchain}

La información contenida en contratos inteligentes se codifica en una base de datos interconectados conocida como blockchain o "cadena de bloques". Existe una inmensidad de definiciones para el blockchain, sin embargo, se lo puede describir como "un sistema descentralizado, cripto-ledger validado por pares que proporciona un público visible, registro cronológico y permanente de todas las transacciones anteriores” (Mik, 2017, p. 6). Como se mencionaba anteriormente el sistema blockchain es una base de datos en la que cada entrada de información es un bloque de información, cada bloque se va interconectando con el anterior y posterior al mismo. Esta interconexión crea una cadena de las entradas de información, o bloques, lo que elimina intermediarios en el proceso de generación de bases de datos, este sistema al ser una interconexión de información, permite mantener los datos ordenados de manera cronológica, inalterable y de manera permanente.

La cadena de bloques emula a un libro de registro en el que quedan grabados los términos legales pactados en los contratos inteligentes para que los mismos se ejecuten de forma automatizada. Así, por medio del blockchain se almacena toda la información de los usuarios y sus movimientos, quedando estos registrados de forma permanente e inmutable; es decir, todas las acciones ejecutadas se pueden visualizar en cualquier momento (Mik, 2017, p. 6). Esta característica brinda seguridad y transparencia a las transacciones, precautelando la integridad de la información.

Los contratos inteligentes, al formar parte del sistema disruptivo de blockchain ofrece grandes beneficios a la administración pública debido a sus características distintivas. Dentro de las principales características podemos nombrar las siguientes: transparencia, irrevocabilidad, eficiencia en tiempo y economía (Porxas \& Conejero, 2018, p. 28). En lo que a transparencia respecta, al estar complementada con el mecanismo de cadena de bloques todos los usuarios tienen la capacidad de visualizar la información que se ha agregado según los movimientos realizados, lo cual impide que se produzcan fraudes al intentar cambiar la información. A su vez, las transacciones realizadas son permanentes debido a que la información que se agrega se distribuye por los nodos que conforman las cadenas de bloques. Es así como los smart contracts presentan soluciones únicas para la administración tributaria, temática que se aborda a continuación.

\subsection{Smart contracts en la administración tributaria}

Por las características antes presentadas, los smart contracts presentan beneficios inigualables para la modernización y eficiencia de la administración tributaria. La recaudación de tributos precisa ser eficiente, para así dar cumplimiento a objetivos de política fiscal, conformando así el presupuesto general del Estado para que su inversión influya positivamente en la sociedad (Garzón, 2018). Por tanto, la eficacia y eficiencia de este sistema permite el éxito económico estatal. Los smart contracts y el blockchain presentan entonces una alternativa para el sistema tributario y la administración pública, ya que estos posibilitan las transacciones ágiles, verificables, seguras y transparentes. 
$\mathrm{Al}$ aplicar los contratos inteligentes en el sistema tributario se presentan beneficios innumerables para la administración pública en la era digital. La seguridad y validación de la información están garantizadas gracias al "sistema de libros de contabilidad donde las transacciones se registran de manera descentralizada creando una pista de auditoría transparente prácticamente inmune a la corrupción y falsificación” (Meeting \& Series, 2017, p. 4). Así también, la ejecución automática de los contratos permite el pago de impuestos de inmediato. De forma automática se realizaría el descuento de fondos de las cuentas bancarias, según se ha preestablecido en el contrato, excluyendo así la posibilidad de que se retrase o no se realice el pago del impuesto. En cuanto a eficiencia, al realizarse un trámite tributario se invierte mucho tiempo en recopilar y verificar datos de deudores. Los contratos inteligentes podrían sintetizar los procesos de validación de datos, también "podría hacer posible activar y liquidar transacciones de forma inmediata, como pueda ser en contratos de seguros o en pagos por resultados" (Grant Thornton, 2018, p. 3).

La imposibilidad de manipulación de la información podría disminuir o eliminar casos de corrupción en la administración tributaria. Ya que los smart contracts se desarrollan en el sistema blockchain este "puede considerarse un registro incorruptible de todas las transacciones anteriores, como una fuente de verdad visible para todos" (Mik, 2017, p. 7). Los datos en la cadena de bloques no pueden modificarse, por ende, los términos y condiciones del smart contract se ejecutarán acorde a lo pactado y codificado inicialmente. En este punto es importante considerar, como en todos los casos, las problemáticas que podrían generarse en aquellos casos en que el contrato no haya sido desarrollado y codificado acorde a la voluntad de las partes (Giuffrida, 2018, p. 779). Para tales casos el derecho debe brindar soluciones en materia contractual, temática de gran relevancia para futuras publicaciones. Si bien la normativa ecuatoriana, de la mano con los planes de innovación gubernamental propende a la aplicación de sistemas tecnológicos nuevos que rompan con las barreras y solucionen los problemas actuales. Si bien los planes de innovación ecuatorianos dan el marco de implementación de las nuevas tecnologías, no son de obligatorio cumplimiento, y aun menos de obligatoria implementación. Todo esto se lo debe vislumbrar desde una perspectiva social por la brecha tecnológica existente dentro del país que afecta a determinados grupos, aspectos que justamente se ha podido evidenciar con las medidas implementadas por la pandemia del COVID-19, como la resistencia al uso de la firma electrónica o en general por la preferencia de la sociedad de acudir a realizar sus trámites de manera presencial.

Es también elemental considerar los beneficios a la interoperabilidad del sistema de administración pública que pueden conllevar los smart contracts. Manejar sistemas coordinados de cadenas de bloques en la administración pública central potenciaría la coordinación de acciones entre las administraciones tributarias locales e incluso internacionales. También se debe considerar la optimización en la relación entre la administración tributaria y el contribuyente, con lo que se transparentan los procesos y se gana la valiosa confianza de los ciudadanos en el sistema estatal.

Debemos también enfatizar el beneficio económico y sistémico que presenta la supresión del uso de intermediarios para realizar transacciones de pago de tributos. Al operativizar el sistema mediante cadenas de bloques y contratos inteligentes, las transacciones se automatizan, eliminando los formularios engorrosos, los intermediarios, y, por ende, los costes de transacción derivados de dichas actividades se excluirían, volviendo así a la tributación directa e instantánea. Es decir, se eliminarían por completo los formularios, pues los mismos estarán codificados y bastará con brindar algunos datos clave y posteriormente firmar de forma electrónica, para que se ejecuten las obligaciones tributarias. 
Toda nueva tecnología presenta retos, sin embargo, la aplicación de los contratos inteligentes en materia tributaria presenta más beneficios que problemáticas. Las alternativas que brinda el sistema de criptografía y los smart contracts dan paso al progreso y eficacia del sistema de administración pública. Los avances representan desarrollo y la palpable era digital exige que la administración pública de pasos fehacientes para un gobierno electrónico en el que las transacciones sean verificables, transparentes, agiles y eficientes. Evidentemente hasta el momento, no se han implementado tecnologías disruptivas que en realidad faciliten la atención a usuarios de forma automatizada, por esto, la ejecución de smart contracts mediante blockchain presenta oportunidades únicas de avances para la sociedad.

\section{Régimen tributario ecuatoriano}

El correcto funcionamiento y cumplimiento de los objetivos del Estado depende, en gran medida, del Presupuesto del Gobierno Central, es así que es prioridad estatal reforzar los mecanismos de recaudación y adaptarlos a las necesidades de una sociedad en la era digital. Uno de los componentes de este presupuesto son los valores recaudados bajo el concepto de tributos. En el Ecuador la regulación del régimen tributario se compone de normativa dispersa. El conjunto de normas principales que rigen el régimen tributario son la Constitución de la República del Ecuador (CRE), el Código Tributario, el Código Orgánico de la Producción, Comercio e Inversiones, la Ley de Régimen Tributario Interno y su reglamento, la Ley Reformatoria para la Equidad Tributaria en Ecuador, el Código Orgánico de Organización Territorial, Autonomía y Descentralización, y todas las ordenanzas que dictan los Gobiernos Autónomos descentralizados dentro de sus competencias.

A pesar de la normativa dispersa, la CRE define, en su política económica las generalidades del régimen tributario. Principalmente, se menciona sobre los principios por los que se regirá el régimen tributario, y que promoverá la política tributaria del país (CRE, art. 300). Sin embargo, los principios constitucionales deben efectivizarse en la administración pública diaria, y el ineficiente funcionamiento de su sistema coarta la garantía o cumplimiento de los derechos y mandatos constitucionales, frente a las necesidades que presenta una sociedad digital.

Es menester también mencionar la complejidad normativa que existe por detrás del sistema tributario, lo cual solamente deteriora la confianza de los ciudadanos en la administración central. A continuación, se presenta un breve contexto del régimen legal tributario en el país, mismo que servirá de pauta para continuar el análisis que compete a la presente investigación.

$\mathrm{Al}$ querer aplicar la figura de los smart contracts en el régimen tributario se precisa entender que la norma tributaria no puede observarse o aplicarse de manera aislada. En este sentido en la legislación ecuatoriana con la vigencia del actual código de comercio ya se conceptualizan y regulan en breves rasgos los contratos inteligentes. Al respecto, la normativa ecuatoriana se menciona de la siguiente manera en el art. 77 del Código de Comercio que dice:

Son contratos inteligentes los producidos por programas informáticos usados por dos o más partes, que acuerdan cláusulas y suscriben electrónicamente.

El programa de contrato inteligente permite facilitar la firma o expresión de la voluntad de las partes, así como asegura su cumplimiento, mediante disposiciones instruidas por las partes, que pueden incluso ser cumplidas automáticamente, sea por el propio programa, o por una entidad financiera u otra, si a la firma del contrato las partes establecen esa disposición. Cuando se dispara una condición pre-programada por las partes, no sujeta a ningún tipo de valoración humana, el contrato inteligente ejecuta la cláusula contractual correspondiente. 
A falta de estipulación contractual, los administradores de dicho programa o quienes tengan su control, serán responsables por las obligaciones contractuales y extracontractuales que se desprendan de los contratos celebrados de esta forma, y en todo caso serán aplicables las disposiciones que protegen los derechos de los consumidores (Código de Comercio, 2019, art. 77).

Se evidencia que entonces la administración tributaria de Ecuador tiene la potestad de valerse de la normativa contractual ecuatoriana para implementar smart contracts en sus procesos de recaudación.

\subsection{Administración tributaria}

El régimen tributario se lo ejecuta a través de la administración tributaria. La administración tributaria se compone de una serie de órganos y funciones que están dotados de competencias para actuar en el "marco de relaciones jurídicas existentes entre el Estado y los contribuyentes" (Parra, 2010, p. 11). Las competencias de cada órgano encargado de la administración tributaria, su creación y facultades deberá ser mediante ley; es decir, dentro de un ámbito legal. En ese sentido el régimen tributario puede ejercerse a través de distintas clases de administración tributaria: la central, la seccional, y la de excepción.

Respecto a la organización de la administración tributaria, destacamos que la misma se delimita territorialmente. Hablamos de administración central, cuando nos referimos a la que compete al ámbito nacional; es decir, gobierno central. En el ámbito seccional, la administración corresponde a los Gobiernos Autónomos descentralizados. Finalmente, se hace referencia a la administración tributaria de excepción, misma que se da cuando la ley de manera expresa concede la gestión tributaria a la propia entidad pública acreedora de tributos. (Código Tributario, Arts. 64, 65 y 66). Entre las formas de administración tributaria mencionadas en conjunto componen el sistema tributario ecuatoriano.

La administración tributaria central encuentra su desarrollo en el Código Tributario, en Ley de Régimen Tributario Interno y su respectivo reglamento. La actividad de la administración tributaria central recae de manera estricta en referencia a los tributos considerados como nacionales, que están encaminados a financiar el presupuesto general del Estado y su dirección pertenece al poder ejecutivo (Código Tributario, art. 64).

Por otro lado, en caso de la administración tributaria seccional, cada Gobierno Autónomo Descentralizado como autoridad tributaria dentro de sus competencias, expide su propia normativa sobre la creación (CRE, art. 264), determinación y recaudación de tributos. (Código Orgánico de Organización Territorial, Autonomía y Descentralización, art. 6).

En el presente estudio nos limitaremos a hablar exclusivamente de la administración tributaria central. Esto debido a que la era digital precisa de respuestas nacionales frente a la administración tributaria, las que, a su vez, influenciarán las decisiones y actuaciones de gobiernos locales.

\subsection{Administración tributaria central y sus facultades}

La administración tributaria central se encarga de ejercer sus facultades respecto de los tributos de carácter nacional. Es así que el órgano competente de la administración tributaria central no aduanera es el Servicio de Rentas Internas (SRI). El presente trabajo se enfoca exclusivamente en la administración tributaria central en lo que refiere a los tributos nos aduaneros recaudados por el SRI. 
Como se mencionaba anteriormente las administraciones tributarias poseen distintas facultades, en el presente trabajo la facultad que nos atańe analizar es la recaudadora. La facultad de recaudación es la que permite a la administración tributaria el cobro de las obligaciones que han nacido de la obligación tributaria, "se efectuará por las autoridades y en la forma o por los sistemas que la ley o el reglamento establezcan para cada tributo." (Código Tributario, art. 71). Es así que se puede entender a la recaudación tributaria como aquella que permite generar ingresos públicos a través de la exigencia de los tributos, establecidos de forma unilateral por el Estado a consecuencia del poder fiscal, conferido por su respectivo ordenamiento jurídico (Torres, 2008, p. 43). El presente trabajo investigativo se centra exclusivamente en la facultad recaudadora de la administración tributaria y los efectos de los smart contracts en la misma, esto debido a que metodológicamente se busca indicar las oportunidades que la tecnología blockchain, aplicada en contratos inteligentes, genera para la recaudación inmediata de tributos por medio de transacciones automatizadas.

La facultad recaudadora de la administración tributaria central debería regularse respecto de cada tributo en la Ley de Régimen Tributario Interno. Al respecto, en el capítulo X de la Ley de Régimen Tributario Interno (LRTI) se establece el sistema para distribuir la recaudación del impuesto a la renta, caso en el que "el producto del impuesto a la renta se depositará en la cuenta del Servicio de Rentas Internas que, para el efecto, se abrirá en el Banco Central de Ecuador" (LRTI, art. 51). Es decir, dentro de este cuerpo normativo no existe una regulación sobre la recaudación tributaria per se, únicamente se define el destino de los montos cobrados.

Por otro lado, sobre el órgano competente para ejercer esta facultad recaudadora encontramos lo establecido en la Ley de creación del Servicios de Rentas Internas (LSRI). Dicho cuerpo normativo en su art. 2 nro. 2, le faculta al SRI en calidad de autoridad tributaria a "Efectuar la determinación, recaudación y control de los tributos internos del Estado." Pero, esta facultad es exclusiva de los tributos internos del Estado, y de aquellos cuya administración no se haya asignado de manera expresa por Ley a otra autoridad.

El SRI es el órgano competente para recaudar los tributos dentro de la administración tributaria central. Dentro de ese sistema existen determinadas competencias especiales que se le reconocen entorno a la recaudación tributaria. Ejemplo de esto es lo regulado en el art. 112 de la LRTI, mismo que establece la posibilidad de la administración tributaria de "celebrar convenios especiales con las instituciones financieras establecidas en el país, tendentes a recibir la declaración y la recaudación de los impuestos, intereses y multas por obligaciones tributarias." (LRTI, art. 112).

Al existir la posibilidad de convenios con instituciones financieras para que sean recaudadoras de impuestos, existe una sanción por el no depósito de los valores que estos organismos recauden. Ya realizada la recaudación por la institución financiera, si a la consignación de este valor no se consigna dentro de los plazos establecidos, se generarán intereses liquidados diariamente, sobre el monto exigible no consignado oportunamente (LRTI, art. 113). Es decir, en el Ecuador existe la intermediación financiera para poder efectivizar el pago de impuestos.

\subsection{Sujetos de la relación tributaria}

Las relaciones jurídicas tributarias se conforman por la interacción de sujetos, y los denominamos "sujetos de la relación tributaria". Dentro del ámbito tributario, los sujetos de la relación son el sujeto activo y el sujeto pasivo. El sujeto activo es el ente público acreedor del tributo (Código Tributario, art. 23) y el sujeto pasivo es "la persona natural o jurídica que, según la ley, está obligada al cumplimiento de la prestación tributaria, sea como contribuyente 
o como responsable" (Código Tributario, art. 24), en este grupo de sujetos pasivos tienen la calidad de tal las herencias yacentes, comunidades de bienes y demás "entidades que, carentes de personalidad jurídica, constituyan una unidad económica o un patrimonio independiente de los de sus miembros, susceptible de imposición” (Código Tributario, art. 24).

A partir de la facultad recaudadora, existen otros sujetos que forman parte de la relación tributaria siendo estos los agentes de retención o percepción. Estos sujetos o agentes también podrán efectuar la recaudación de tributos, en caso de que la ley lo establezca o que instituya la administración. (Código Tributario, art. 71). Por agentes de retención se entienden a las personas naturales o jurídicas que "en razón de su actividad, función o empleo, estén en posibilidad de retener tributos y que, por mandato legal, disposición reglamentaria u orden administrativa, estén obligados a ello" (Código Tributario, art. 29). Son también agentes de retención los herederos y albaceas respecto de los impuestos que se deban cancelar por concepto de herencias y legados.

Los otros sujetos en la relación tributaria son aquellos a los que la ley les permite recaudar tributos, también llamados "agentes de percepción". Son agentes de percepción las personas naturales o jurídicas que por la actividad, función o empleo que realizan, y que, por mandato legal o reglamentario, están obligados a recaudar tributos y entregarlos al sujeto activo.

\subsection{Exigibilidad para la recaudación tributaria}

Existe una relación intrínseca entre todas las facultades de la administración tributaria. La facultad de determinación y la de recaudación tienen su punto de encuentro en que la recaudación tributaria necesita un acto de determinación para que se realice o se haga efectiva. Esta determinación se la cumple a través de las declaraciones que las realizan los mismos contribuyentes. En esta misma línea de actos de determinación, encontramos a los actos administrativos de determinación que tengan la característica de firmes o ejecutoriados, y de la misma manera, por medio de sentencias ejecutoriadas u "otros hechos o actos preestablecidos en forma legal, que contengan obligaciones determinadas líquidas y convertidas en títulos de crédito" (Parra, 2010, p. 17). Estos títulos de crédito deben llevar de manera implícita una orden de cobro, es importante mencionar que el procedimiento por el cual se emitirán los mismos no puede estar pendiente de resolución o existir algún recurso que aún se pueda interponer (Código Tributario, art. 149).

No siempre los sujetos pasivos cumplen con sus obligaciones tributarias de la manera esperada. En caso de que esto suceda; es decir, la recaudación no haya cumplido con su objetivo y los sujetos pasivos no hayan pagado su obligación a través de los órganos regulares, la administración tributaria cuenta con la jurisdicción coactiva para hacer efectivos dichos créditos. Quien puede ejercer la acción coactiva, es decir el órgano competente recae en los funcionarios recaudadores de las administraciones tributarias; y las máximas autoridades pueden designar recaudadores especiales para ejercer la acción coactiva en las secciones territoriales que consideren necesario (Código Tributario, art. 158)

Con el presente análisis jurídico se logra contextualizar al lector respecto de la funcionalidad del régimen tributario en lo que al sistema de recaudación en la normativa ecuatoriana compete. La normativa es bastante dispersa y se desarrolla en tantos cuerpos normativos que resulta complejo su manejo, sin mencionar su continua modificación mediante Leyes de carácter económico urgente. De igual manera, se puede evidenciar la falta de cohesión entre normas tributarias, esto se debe a la falta de claridad en el régimen de competencias de las distintas administraciones tributarias. El ordenamiento jurídico ecuatoriano en materia tributaria carece de organización, y se define en una inmensidad de reglamentos que impiden un efectivo estudio de este. Para implementar nuevos sistemas que propendan modernizar el 
sistema tributario y la recaudación tributaria nacional, será preciso observar las normas implícitas y consolidar normativa clara, concisa que permita desarrollar sistemas informáticos efectivos que transparenten la administración tributaria a las normas que la legitiman.

\subsection{Intentos en el sistema tributario ecuatoriano para pasar a lo digital}

La administración tributaria ha tenido algunos intentos con miras a la modernización digital de la misma. Con el objetivo de facilitar el servicio en línea y la accesibilidad de todos los sujetos pasivos o contribuyentes que no podían acceder con facilidad a los formularios de tributación, o no disponían del tiempo para ajustarse a horarios de oficina, varios procedimientos del sistema tributario se han digitalizado. Un claro ejemplo de esto es la resolución NAC-DGERCGC17-00000032 (Resolución 032) mediante la que el SRI dispone la implementación del nuevo Portal Digital operativo los 365 días del año y con bases de datos explicativas de cada procedimiento.

En este portal se puede encontrar:

- Servicios en línea

- Trámites electrónicos

- Atención telefónica

- Cita previa para las agencias de recaudación de impuestos

- Catálogos, guías y manuales de explicación de procedimientos.

La Resolución 032 también plantea la obligatoriedad de los servicios en línea para acceder a ciertos trámites ciudadanos. Los trámites que cuentan con esta característica de obligatoriedad son todos aquellos referentes al RUC, certificados automáticos con código QR, y, formularios y certificaciones identificadas (Resolución 032, art.1). Este claro esfuerzo de digitalizar las funciones más sencillas y que pueden ser realizadas de forma autónoma, es un ejemplo concreto de la necesidad de modernizar el sistema tributario nacional. Ejemplo de esto son aspectos como los trámites que se pueden realizar en la plataforma del SRI como la actualización del RUC, la solicitud para emitir facturas electrónicas, la presentación de solicitudes de inscripción de RUC, el pago de tributos, entre otros trámites que ya se pueden realizar de manera digital en su integralidad.

Para el uso del sistema existen determinados requisitos que continúan entorpeciendo la administración tributaria. Para cumplir con los mismos existen acciones desde la generación de usuarios y claves, hasta la obtención del Registro Único de Contribuyentes. Incluso, para acceder a todos los servicios en línea del portal "los contribuyentes deberán contar con la clave de uso de medios electrónicos para el acceso a los servicios brindados a través del portal web del Servicio de Rentas Internas" (Resolución 032, art.2). Sin embargo, enfrenta nuevamente la problemática planteada, ¿̇a dónde van a parar estos folios digitales? Es preciso entonces un sistema que brinde transparencia y agilidad en las transacciones online.

Existe otro régimen tributario importante de análisis respecto de la recaudación en términos de modernización. Nos referimos al Régimen Impositivo Simplificado que se encuentra establecido en la Ley Reformatoria para la Equidad Tributaria en el Ecuador. Este régimen se les aplica a los contribuyentes que cumplen con las características legales establecidas para que puedan pagar sus cuotas mensuales con dinero electrónico caso en el que obtienen un descuento de pago a cuota, equivalente al 5\% de la misma. (Resolución NAC-DGERCGC16-00000238 SRI, art. 2.). Este régimen se creó con el objetivo de que los contribuyentes pasen a formar parte de este nuevo sistema electrónico con miras a una simplificación y celeridad dentro del proceso de recaudación. 
Se evidencian esfuerzos para modernizar la administración tributaria central; sin embargo, no hay intentos claros de definir un sistema que realmente agilice las transacciones y permita la descentralización de información que brinde transparencia a usuarios.

\subsection{Necesidad de digitalización del Sistema tributario en el marco de la pandemia del COVID-19.}

El inicio de contagios por COVID-19 dentro del Ecuador puso de manifiesto una serie de problemáticas gubernamentales dentro de muchos aspectos del Estado, como educación, salud, economía, entre otros. Como medida principal para evitar aglomeraciones de personas en un mismo espacio para evitar contagios masivos, el sistema burocrático del país debió tomar alternativas para los trámites necesarios de la ciudadanía. La administración tributaria no fue la excepción y se vio en la necesidad de implementar medidas digitales para que los contribuyentes pueden seguir realizando los trámites y cumpliendo con sus obligaciones tributarias.

El SRI el 30 de marzo firmó la Resolución Nro. NAC-DGERCGC20-00000023 (Resolución 023 en adelante), la que se promulgó en el Registro Oficial el 20 de abril del presente año. En esta resolución se define un sistema para la recepción de documentos por medios electrónicos y la gestión interna de los mismos, durante el tiempo del estado de excepción.

La resolución 023 da los lineamientos generales a ser tomados respecto de las comunicaciones, consultas, peticiones, solicitudes y requerimientos dirigidos al SRI. Cabe señalar que dicho marco legal solo regula el uso del Portal, sistematiza determinados procesos para la situación de emergencia en contexto COVID-19. En este sentido, la documentación presentada ante el SRI se puede realizar a través del sistema documental Quipux y de correo electrónico. Dentro del sistema Quipux la información presentada deberá contar con firma electrónica (Resolución 023, art. 3 nro.1), mientras que las peticiones enviadas a través de correo electrónico se deberán remitir en formato PDF, firma autógrafa del peticionario y con la expresa declaración sobre la presentación física de los documentos una vez terminado el estado de excepción (Resolución 023, art. 3 nro. 2). A continuación, presentamos un gráfico de la sistematización de cómo se llevarán de manera interna estos procesos a través de medios electrónico:

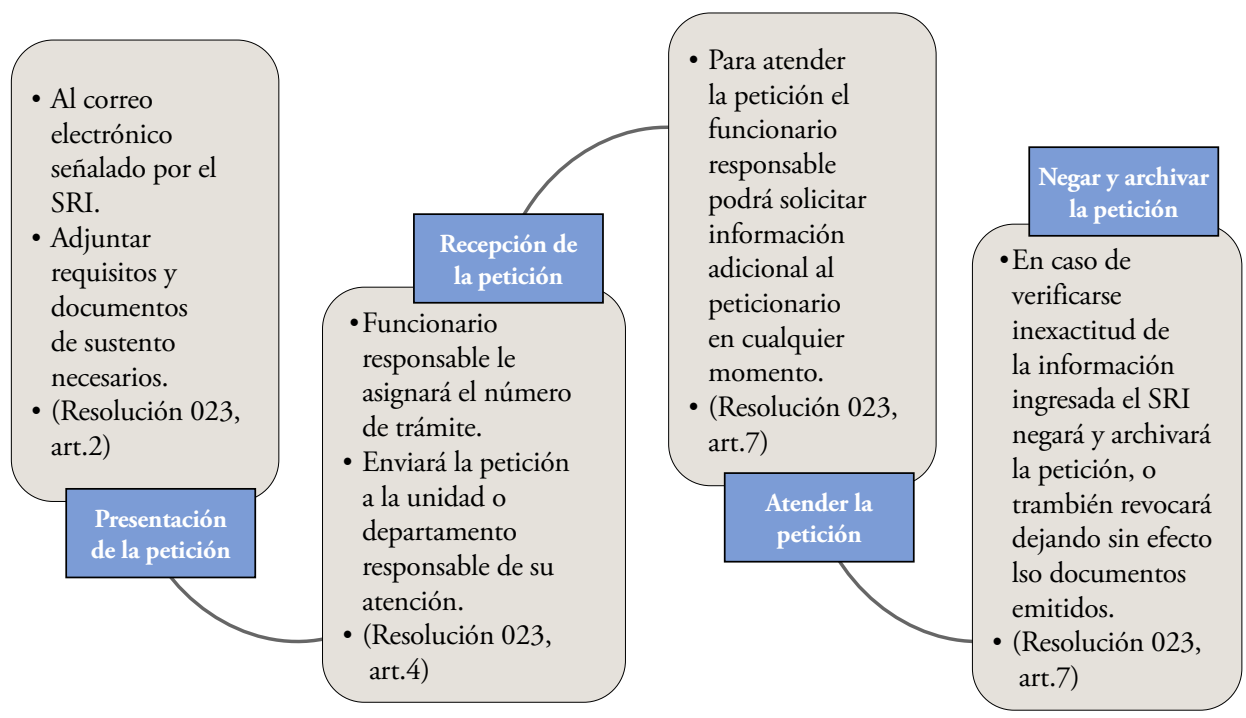

Figura 2. Proceso SRI en contexto COVID -19. Gráfico realizado con base a la Resolución Nro. NAC-DGERCGC20-00000023, expedida por el SRI. 
Mencionadas las medidas tomadas por la administración tributaria central, con miras a la modernización de sus servicios es necesario analizar las mismas. Tanto el portal de servicios del SRI, como el régimen simplificado (RISE) y la reglamentación para que se reciban los documentos por medios electrónicos, son políticas tomadas con miras a una digitalización de la información y a procesos más expeditos. Si bien estas opciones dan lugar a posibilidades de recaudación más sencillas, como el pago en línea, las mismas no comprenden una solución efectiva que evite fraudes y dilaciones de pagos, brindando garantizando la seguridad y veracidad de la información que se remite. Es decir, la implementación de medidas para digitalizar los servicios de recaudación tributaria solo reafirma la necesidad de potenciar la administración tributaria mediante sistemas distintivos que democraticen el sistema, brindando real transparencia a las transacciones, como lo son los smart contracts por medio de blockchain.

\section{Modernización de la recaudación tributaria por medio de smart contracts en Ecuador}

La epidemia del COVID-19, con la cual ya no es posible hacer trámites físicos, pone de manifiesto la necesidad de contar con medios tecnológicos que permitan desarrollar trámites de administración pública por medios electrónicos. Incluso posterior a la etapa de COVID-19, la humanidad no estará lista para regresar a sistemas obsoletos; es decir, la implementación de nuevas tecnologías debe ser progresiva, es entonces necesario considerar seriamente al blockchain y los smart contracts que presentan una gran opción para la recaudación de impuestos centrales, seccionales y aduaneros. A continuación, un recuento respecto a las problemáticas, beneficios y necesidades de implementar estas tecnologías en el Ecuador.

\subsection{Problemáticas del sistema tributario ecuatoriano actual}

En los puntos anteriores se han analizado las diferentes potestades que ejerce el sistema tributario central en ese sentido, atañe al presente trabajo estudiar las dificultades que presenta la administración de un aparato tan complejo. En esta línea, se debe comprender que un aparataje tan extenso, de orden jerárquico, representa también una línea de tramitación larga y compleja. Es decir, el principal problema que presenta el aparataje tributario es la complejidad y falta de claridad en la aplicación de su normativa que repercute en complicaciones pragmáticas del sistema. Este entramado de procedimientos y formularios necesarios para tributar, problematizan la recaudación tributaria debido al desconocimiento de la sociedad (López, 2017, p. 4). Los continuos cambios normativos y su falta de socialización convergen en un sistema desorganizado que solo da complicaciones a sus usuarios.

Debido a que el sistema tributario responde a una economía global, los efectos problemáticos de la normativa nacional también encuentran su sustento a la participación de capitales extranjeros. A continuación, se presentan dos tablas que resumen las problemáticas a nivel nacional e internacional del sistema tributario ecuatoriano. El primero, describe las aquellos inconvenientes identificados a nivel nacional, en relación con las dificultades con quienes están llamados a tributar (sujetos de la tributación), los procedimientos de recaudación, capacitación interna y gasto tributario que realiza el Estado. Por otro lado, la segunda tabla resume las consecuencias de que temas globales como inflación, doble imposición internacional, deuda externa tienen en la administración tributaria del Ecuador. 


\begin{tabular}{|c|c|c|c|c|c|}
\hline ÁREA & \multicolumn{5}{|c|}{ ORDENAMIENTO TRIBUTARIO NACIONAL } \\
\hline Problemática & $\begin{array}{l}\text { Sistema de } \\
\text { recaudación }\end{array}$ & $\begin{array}{l}\text { Personas } \\
\text { llamadas a } \\
\text { tributar }\end{array}$ & $\begin{array}{l}\text { Procesos de } \\
\text { tributación }\end{array}$ & $\begin{array}{l}\text { Capacitación } \\
\text { para usuarios }\end{array}$ & $\begin{array}{c}\text { Gasto } \\
\text { Tributario }\end{array}$ \\
\hline \multirow[t]{2}{*}{$\begin{array}{l}\text { Detalle del } \\
\text { problema }\end{array}$} & $\begin{array}{c}\text { Excesivo } \\
\text { número de } \\
\text { trámites } \\
\text { administrativos } \\
\text { existentes } \\
\text { complican el } \\
\text { dinamismo y } \\
\text { generan más } \\
\text { burocracia }\end{array}$ & $\begin{array}{c}\text { El sistema } \\
\text { infunde } \\
\text { temor en sus } \\
\text { contribuyentes } \\
\text { a través de } \\
\text { sanciones } \\
\text { pecuniarias lo } \\
\text { que constituye } \\
\text { un rechazo del } \\
\text { mismo }\end{array}$ & $\begin{array}{l}\text { Los procesos } \\
\text { se encuentran } \\
\text { reglados } \\
\text { por la } \\
\text { codificación } \\
\text { tributaria } \\
\text { compleja de } \\
\text { analizar y de } \\
\text { difícil acceso. }\end{array}$ & $\begin{array}{l}\text { La capacitación } \\
\text { en el sistema } \\
\text { tributario sobre } \\
\text { sus portales } \\
\text { en línea se da } \\
\text { exclusivamente a } \\
\text { los funcionarios } \\
\text { que están en } \\
\text { contacto con el } \\
\text { mismo, haciendo } \\
\text { inefectiva la } \\
\text { atención directa } \\
\text { al cliente, por lo } \\
\text { que se necesita } \\
\text { una capacitación } \\
\text { continua a la } \\
\text { externa de las } \\
\text { instituciones } \\
\text { dirigida a los } \\
\text { usuarios del } \\
\text { sistema. }\end{array}$ & $\begin{array}{l}\text { La creación } \\
\text { de subsidios } \\
\text { para ciertos } \\
\text { sectores de } \\
\text { la economía } \\
\text { nacional } \\
\text { perjudica la } \\
\text { recaudación } \\
\text { de tributos } \\
\text { generando } \\
\text { un déficit en } \\
\text { el ingreso de } \\
\text { capital. }\end{array}$ \\
\hline & & $\begin{array}{l}\text { Los trámites } \\
\text { son complejos } \\
\text { con trabas } \\
\text { engorrosas }\end{array}$ & & & \\
\hline $\begin{array}{l}\text { Solución } \\
\text { por Smart } \\
\text { Contracts }\end{array}$ & $\begin{array}{l}\text { Procesamiento } \\
\text { de trámites } \\
\text { de forma } \\
\text { automatizada } \\
\text { mediante } \\
\text { código binario, } \\
\text { sin exceso } \\
\text { de revisión } \\
\text { gramatical } \\
\text { incide en } \\
\text { agilidad y } \\
\text { mejor uso } \\
\text { de recursos, } \\
\text { evitando } \\
\text { burocracia }\end{array}$ & $\begin{array}{l}\text { Las herramient } \\
\text { hacen el sister } \\
\text { amigable a }\end{array}$ & $\begin{array}{l}\text { s tecnológicas } \\
\text { a accesible y } \\
\text { s usuarios }\end{array}$ & $\begin{array}{c}\text { Simplificación } \\
\text { del sistema en } \\
\text { relación con los } \\
\text { procesos, para } \\
\text { dar efectividad al } \\
\text { mismo. }\end{array}$ & $\begin{array}{l}\text { Optimización } \\
\text { del sistema } \\
\text { actual } \\
\text { relacionado } \\
\text { con la } \\
\text { transparencia } \\
\text { en } \\
\text { seguimiento } \\
\text { de deudores }\end{array}$ \\
\hline
\end{tabular}

Tabla 1: Problemáticas nacionales (Jurow, 2020, p. 47). 


\begin{tabular}{|c|c|c|c|c|}
\hline ÁREA & \multicolumn{4}{|c|}{$\begin{array}{c}\text { FACTORES EXTERNOS CON INJERENCIA EN } \\
\text { EL SISTEMA TRIBUTARIO NACIONAL }\end{array}$} \\
\hline Problemática & Inflación & $\begin{array}{c}\text { Doble } \\
\text { imposición } \\
\text { internacional }\end{array}$ & Riesgo país & $\begin{array}{l}\text { Empresas con } \\
\text { sucursales en el } \\
\text { extranjero }\end{array}$ \\
\hline $\begin{array}{l}\text { Detalle del } \\
\text { problema }\end{array}$ & $\begin{array}{l}\text { Aumento de precios } \\
\text { en los principales } \\
\text { sectores comerciales que } \\
\text { derivan en el menor } \\
\text { flujo económico y el } \\
\text { incremento del gasto } \\
\text { público }\end{array}$ & $\begin{array}{l}\text { Dos o más } \\
\text { jurisdicciones } \\
\text { tributarias } \\
\text { pueden exigir } \\
\text { para sí el } \\
\text { gravamen de } \\
\text { determinada } \\
\text { renta. }\end{array}$ & $\begin{array}{c}\text { La poca } \\
\text { efectividad de } \\
\text { los procesos } \\
\text { tributarios } \\
\text { nacionales } \\
\text { genera } \\
\text { incertidumbre } \\
\text { y desconfianza } \\
\text { a las inversiones } \\
\text { extranjeras. }\end{array}$ & $\begin{array}{l}\text { No se norma el } \\
\text { ingreso bruto } \\
\text { generado por la } \\
\text { actividad efectiva } \\
\text { desarrollada en el } \\
\text { extranjero }\end{array}$ \\
\hline $\begin{array}{l}\text { Solución } \\
\text { por Smart } \\
\text { Contracts }\end{array}$ & $\begin{array}{l}\text { Procesos transparentes } \\
\text { que permiten tomar } \\
\text { decisiones en tiempo } \\
\text { real. Beneficios a } \\
\text { captación de tributos } \\
\text { y mayor claridad en } \\
\text { información para } \\
\text { procesos coactivos. }\end{array}$ & $\begin{array}{c}\text { Registro } \\
\text { automático } \\
\text { permanente } \\
\text { de las } \\
\text { transacciones } \\
\text { del } \\
\text { contribuyente, } \\
\text { que brinda } \\
\text { transparencia. }\end{array}$ & $\begin{array}{r}\text { La plataforma } \\
\text { información en la } \\
\text { smart contracts evi } \\
\text { de un potenc } \\
\text { transparentan } \\
\mathrm{p}\end{array}$ & $\begin{array}{l}\text { de verificación de } \\
\text { que se desarrollan los } \\
\text { a el desconocimiento } \\
\text { al contribuyente, } \\
\text { lo la información } \\
\text { blica, }\end{array}$ \\
\hline
\end{tabular}

Tabla 2: Problemáticas generadas en contexto de globalidad (Vallejo y Maldonado, 2007, pp. 39-59).

Frente a los problemas presentados se evidencia la necesidad de que los procesos se agiliten y puedan descargar y optimizar el sistema tributario nacional. Se debe comprender que las falencias derivan de la carga de trabajo y el sistema de archivos físicos por medio de folios, el que resulta ineficaz no solo para la recaudación de tributos que ya es de cierta manera electrónica, sino para todos los procesos tributarios que se realizan. Las problemáticas presentadas evidencian la urgente necesidad de que la administración pública se valga de nuevas tecnologías para optimizar sus servicios en pro de la sociedad.

Para que se evidencie la necesidad que representa encontrar un sistema efectivo y rápido de procesamiento de información, se debe recordar el fin último del sistema tributario. En base a esto cabe plantear la interrogante de cuál es la situación que entorpece el sistema tributario e impide que alcance este objetivo abstracto para el cual fueron creados los tributos en primer lugar. Como se mencionaba con anterioridad la problemática radica en el número de trámites existentes, de la mano con la complejidad y diversidad normativa existente alrededor del sistema. Si bien los smart contracts pueden ser una solución al primer punto, dentro del sistema tributario la norma debe dar un cambio en su contenido para que dé cabida a estos procesos tecnológicos con enfoque de simplicidad en pro de la administración tributaria.

Ahora bien, esta respuesta fue encontrada décadas atrás por la migración de datos a bases de almacenamiento. En este sentido, el estado ecuatoriano ha realizado diferentes esfuerzos para alcanzar los beneficios de las nuevas tecnologías. 


\subsection{Soluciones que presentan los smart contracts al sistema tributario del Ecuador}

Como se detalla en la parte inicial de este artículo, los smart contracts y el blockchain presentan una herramienta tecnológica que efectiviza el procesamiento y almacenamiento efectivo, eficaz y transparente de la información. El ingreso de datos y el orden efectivo en el que se almacenan agilita tanto al sistema tributario en su labor de captación, como al sujeto pasivo en su papel de contribuyente. Es decir, se identifican de mejor manera las cifras más altas de deuda tributaria y se concentran los recursos de fiscalización en montos efectivos, representativos para la economía versus, lo que sucede actualmente, sobre la coactiva a pequeños deudores (Jurow, 2020, p. 44).

Por medio del sistema blockchain y los smart contracts se generaría una guía confiable, dinámica y auto desarrollada de pasos a seguir para optimizar las transacciones en el sistema tributario. De esta forma, los funcionarios públicos cumplirían con más eficiencia sus funciones sin necesidad de capacitación previa, pues el sistema presentaría soluciones y respuestas (López, 2017, p. 7). La codificación de contratos y uso de códigos para determinar ciertas cláusulas, validando tales transacciones mediante blockchain, permite que optimizar el trabajo de los funcionarios y facilita la revisión de ciertos aspectos que muchas veces pueden confundirse por disyuntivas incluso gramaticales (Jurow, 2020, p. 5).

Debido a que el cambio de paradigma contractual a smart contracts permitiría el almacenamiento y organización de datos de forma remota. Esto incrementa la unidad del ordenamiento tributario para evitar la duplicidad de esfuerzos y falencias en comunicación que resulta en la pérdida de fondos y mala inversión de capitales públicos. Mediante la implementación de smart contracts se digitalizarían los procedimientos, almacenando la información en bases de datos que clasifiquen y archiven automáticamente la información, esto permitiría que el funcionario obtenga respuestas concretas y veloces frente a las necesidades de los contribuyentes.

En el ámbito internacional, la implementación de smart contracts en las transacciones tributarias incrementaría la confiabilidad en la administración pública. La capacitación de los funcionarios, el tema tecnológico y el avance de los paradigmas tributarios tradicionales a un efectivo gobierno electrónico (Harvard Law Review, 2008) genera confianza para inversión extranjera en el país. Esto se debe a la confianza en un sistema tributario que canaliza y posibilita el flujo económico nacional con tecnologías transparentes, disminuyendo pérdidas fiscales y potenciando ingresos.

En conclusión, la implementación de smart contracts, alivia la cantidad de información del sistema tributario y por tanto no asfixia ni a los funcionarios, ni a los contribuyentes, con la compleja estructura de presentación de documentos y certificaciones (Hoffman, 2018, p. 2). Por el contrario, hace efectiva la relación sistema-sociedad, de tal forma que el contribuyente sienta confianza de su relación con el fisco, enriqueciendo así la cultura tributaria nacional.

\subsection{Necesidad de modernizar el sistema tributario ecuatoriano mediante smart contracts} La consecución de los Objetivos de Desarrollo Sostenible de la Agenda 2030 conlleva a la promoción y refuerzo en el uso de estas tecnologías, y por lo mismo es necesario, según el Informe sobre la Economía Digital del año 2019 (United Nations Conference of Trade and Development, 2019, p. 3), así como en el Infome E- Government realizado por la ONU en el año 2018, la prioridad de implementar políticas estatales para promover una transformación digital (Department of Economic and Social Affairs of the United Nations Secretariat. 2019).

El Ministerio de Telecomunicaciones en su Libro Blanco de la Sociedad de la Información y del Conocimiento establece como objetivo del Programa de implementación para la digitalización de las empresas hacia la Transformación Digital: “Mejorar la productividad 
y la competitividad de las industrias del país, a través de la Transformación Digital" (Ministerio de Telecomunicaciones del Ecuador, 2019, p. 68), este programa tiene como líneas de acción "facilitar el entorno hacia la transformación digital en las empresas, fomentar el uso de comercio electrónico, promover el emprendimiento e innovación de base tecnológica, y generar acciones para el desarrollo de la Industria TIC”. Lo que evidencia el interés por parte del gobierno central por impulsar la economía digital en el Ecuador.

El Plan de la Sociedad de la Información y del Conocimiento (PSIC) para el período 2018-2021 busca propiciar el desarrollo nacional a través de programas y proyectos que permitirán alcanzar objetivos trazados en la Política Nacional de Telecomunicaciones y de la Sociedad de la Información, elaborada para el período 2017-2021 (Ministerio de Telecomunicaciones y de la Sociedad de Información, 2017, p. 10). El PSIC en su objetivo 4.3.3 busca la implementación de la tecnología de Registros Distribuidos (Distributed Ledger Technology o blockchain) (Ministerio de Telecomunicaciones y de la Sociedad de Información, 2017, p. 30).

También cabe mencionar la necesidad de involucrar a la academia, a organismos internacionales y organizaciones de la sociedad civil. Estas alianzas permitirán posibilitar la ejecución de proyectos de este tipo, posesionando al país a nivel internacional por los avances en sus sistemas tributarios, que a su vez implicarían mejoras en la dinamicidad de la economía, sistema gubernamental y confianza de los ciudadanos. Es elemental generar alianzas estratégicas para traer a ecuador la evolución de la era digital.

De acuerdo con los datos del Instituto Nacional de Estadística Censos (INEC), el gasto total en ACTI (Actividades de Ciencia, Tecnología e Innovación) entre el 2009 al 2014 incrementó en un 88,92\% (Instituto Nacional de Estadística y Censos, 2014, p. 15). El ACTI está conformado por Investigación y Desarrollo Agregado, Otras Actividades de Ciencia y Tecnología, y Otras actividades de Innovación.

Igualmente, el gasto en Ciencia y Tecnología creció en un $122 \%$ entre los años 2009 y 2014. En la misma línea, el gasto en innovación durante el periodo 2012-2014 en un 74,47\% representa financiamiento del sector privado y asciende a \$3.175,27 millones de dólares (Instituto Nacional de Estadística y Censos, 2014, p. 18).

Gradualmente lo virtual va más allá de la soberanía de países y elimina fronteras, es deber de cada Estado entonces impulsar el uso de TICs para el desarrollo global de su sociedad, así como velar por el progreso de su economía creando oportunidades que van de la mano con la realidad de la cuarta revolución industrial y la era globalizada. Esto va de la mano con la Política 5.6 del Objetivo 5 Eje 2: Economía al Servicio de la Sociedad del Plan Nacional de Desarrollo 2017-2021 (Secretaría Nacional de Planificación y Desarrollo, 2017, p. 83). Ecuador podría aprovechar las inversiones en ingenio a nivel nacional para desarrollar software y hardware destinados a la modernización de la administración tributaria en Ecuador con el uso de smart contracts y tecnología blockchain.

En el país existe efectivamente inversión en innovación y tecnología. Estos fondos podrían ser asignados en gran parte a la modernización de la administración tributaria, que a su vez dinamizaría la economía nacional y atraería inversión extranjera, junto con un sistema confiable y automatizado que satisfaga a sus usuarios. Esto también respondería a necesidades palpables en el contexto de una sociedad digital que ha dado el paso de la modernización en el contexto COVID -19 y no volverá atrás. Tanto la Agenda 2030 como la sociedad moderna exigen del Estado ecuatoriano las respuestas que solo tecnologías disruptivas como los smart contracts y el blockchain pueden dar, queda por definir si el gobierno está dispuesto a atender a necesidades prioritarias. 


\section{Conclusiones}

Las alternativas que brinda el sistema de criptografía y los smart contracts dan paso al progreso y eficacia del sistema de administración pública. Los avances representan desarrollo y la palpable era digital exige que el Estado genere, valiéndose de avances tecnológicos, transacciones verificables, transparentes, ágiles y eficientes.

El ordenamiento jurídico ecuatoriano en materia tributaria carece de organización, y se define en una inmensidad de reglamentos que impiden su efectivo estudio. Para implementar nuevos sistemas que propendan modernizar el sistema tributario y la recaudación tributaria nacional, será preciso observar las normas implícitas y consolidar normativa clara. Si bien se evidencian esfuerzos para modernizar la administración tributaria central, hace falta sistemas que realmente agilicen las transacciones y permitan la descentralización y transparencia de información.

Es preciso que en Ecuador se fomente la confianza en la administración tributaria mediante las nuevas tecnologías disruptivas como el blockchain y smart contracts. Frente a las necesidades de una sociedad digital que ha dado el paso de la modernización y no volverá atrás. Tanto la Agenda 2030 como la sociedad moderna exigen del Estado ecuatoriano las respuestas que solo sistemas como los smart contracts y el blockchain pueden brindar.

\section{Referencias bibliográficas}

Agénor, Pierre-Richard (2001). The Economics of Adjustment and Growth. Academic Press. Ameer, R. (2016, diciembre 20). Smart Contracts: The Blockchain Technology That Will Replace Lawyers. < https://blockgeeks.com/guides/smart-contracts/ >

American Taxpayer Relief Act of 2012, Pub. L. Nro. 112-240

Asamblea Nacional. (1997). Ley de creación del Servicio de Rentas Internas. Quito: Registro Oficial.

Asamblea Nacional del Ecuador. (2005). Código Tributario. Quito: CEP

Asamblea Nacional del Ecuador. (19 de octubre de 2010). Código Orgánico de Organización Territorial, Autonomía y Descentralización. Quito: Registro Oficial Registro Oficial Suplemento. 303.

Asamblea Nacional del Ecuador. (2004). Ley de régimen tributario interno. Quito : CEP

Bustos, J. (2018). El problema no son los impuestos, son los empresarios. Recuperado el 12 de Junio de 2020 de: https:/www.eltelegrafo.com.ec/noticias/punto/1/elproblema-no-son-los-impuestos-son-los-empresarios

Bureau, C. (2015). State \& Local Government Finance Historical Datasets and Tables. $<$ https://www.census.gov/data/datasets/2015/econ/local/public-usedatasets.html [https://perma.cc/ZNG2-52B3]>

Deloitte. (2017). Blockchain technology and its potential in taxes. December $</$ https:// www2.deloitte.com/pl/en/pages/tax/articles/blockchain-technology.html/>

Department of Economic and Social Affairs of the United Nations Secretariat. (2019, enero 01). United Nations E-Government Survey: < https://publicadministration. un.org/egovkb/Portals/egovkb/Documents/un/2020-Survey/2020\%20UN\%20 E-Government\%20Survey\%20(Full\%20Report).pdf >

Digital Chamber of Commerce. (2018). Smart Contracts: Is the Law Ready?. September $</$ https://lowellmilkeninstitute.law.ucla.edu/wp-content/uploads/2018/08/SmartContracts-Whitepaper.pdf/> 
Garzón, M. A. A. P J. (2018). El Sistema Tributario y su impacto en la Economía Popular y Solidaria en el Ecuador. The taxing system and its impact on the Popular and Solidarity Economy in Ecuador. UNIANDES EPISTEME: Revista de Ciencia, Tecnología e Innovación., 5, 38-53.

Giuffrida, I, et al. (2018). A legal perspective on the trials and tributations of AI: How Artificial Intelligence, the Internet of Things, Smart Contracts, and other technologies will affect the law. Case Western Reserve Law Review 68 (3), 747-781.

Harvard Law Review. (2013). Importing a Trade or Business Limitation into \$ 2036: Toward a Regulatory Solution to FLP-Driven Transfer Tax Avoidance. Boston.

Harvad Law Review. (2008). Taxing Private Equity Carried Interest Using An Incentive Stock Option Analogy. Boston.

Hoffman, M. (2018). Can blockchains and linked data advance taxation. 3rd Workshop on linked data \& distributed ledgers. Lyon.

Instituto Nacional de Estadística y Censos. (2014). Principales Indicadores de Actividades de Ciencia, Tecnología e Innovación.

Jiménez, J. (n.d.). Cuestiones jurídicas en torno a la cadena de bloques («blockchain») y a los contratos inteligentes («smart contracts»). Revista Cuatrimestral de Las Facultades de Derecho y Ciencias Económicas y Empresariales, 101.

Jurow, A. (2020). Tax Limits and the Future of local Democracy. Harvard Law Review.

Kraft, J. (2019). Blockchain and Property Rights. Retrieved <https://www.newamerica.org/ future-property-rights/reports/proprightstech-primers/blockchain-and-propertyrights/>

López, D. (2017). El Sistema Fiscal En El Ecuador Y Su Efecto En La Redistribución De La Riqueza. Análisis Entre Los Ańos 2010 - 2015. IAEN.

Meeting, F., \& Series, M. (2017). "Blockchain: Taxation and Regulatory Challenges and Opportunities " by the WU / NET Team. 024(January), 15-16.

Mik, E. (2017). Smart Contracts: Terminology, Technical Limitations and Real World Complexity. Ministerio de Telecomunicaciones del Ecuador. (2019, febrero 10). Libro Blanco de la Sociedad de la Información y del Conocimiento. Retrieved from: < https://www. telecomunicaciones.gob.ec/wp-content/uploads/2019/05/libro-blanco-de-lasociedad-de-la-informacion-y-del-conocimiento..pdf.>

Ministerio de Telecomunicaciones y de la Sociedad de Información. (2017). Políticas Públicas del Sector de las Telecomunicaciones y de la Sociedad de la Información 2017 2021. Quito: Registro Oficial nro. 15.

Parra, R. (2010). La declaración patrimonial en el Ecuador. Quito: Universidad de Cuenca.

Porxas, Núria; Conejero, M. (2018). Tecnología blockchain : funcionamiento, aplicaciones y retos jurídicos relacionados. Actualidad Jurídica Uría Menéndez, 24-36.

Presidencia de la Republica. (2010). Reglamento a la ley de régimen tributario interno. Quito: CEP

ProUniversitarios. (2018, mayo 07). Cómo funciona Blockchain. Retrieved from <http://prouniversitarios.com/como-funciona-blockchain/>

Raskin, M. (2017). The Law And Legality Of Smart Contracts. Georgetown Law Technology Review (305), 205-207.

Secretaría Nacional de Planificación y Desarrollo. (2017). Plan Nacional de Desarrollo 20172021.

Serrano, J. Análisis Dinámico De La Recaudación Tributaria En El Ecuador Aplicando Modelos Varios. 
Torres, M. (2008). Estructura de los ingresos tributarios en el presupuesto general del Estado. Quito: UASB.

United Nations Conference of Trade and Development. (2019). Digital Economy Report. <https://unctad.org/en/pages/PublicationWebflyer.aspx?publicationid=2466>

Villacís, J. (2013). Diseño de una propuesta de plan de capacitación que contribuya al cumplimiento voluntario de las obligaciones tributarias. Quito: Universidad Internacional del Ecuador. 